\title{
Will recent Polish guidelines improve clinical practice in neurology and neurosurgery?
}

Over the last three years, the Polish Journal of Neurology and Neurosurgery (Neurologia i Neurochirurgia Polska) has published four position statements/guidelines regarding different neurological and neurosurgical issues [1-4]. The hope is that these will improve the everyday practice of neurology and neurosurgery in Poland.

The first paper, authored by the Polish Thrombectomy Initiative group and published in 2017, presented the results of a structured questionnaire collected from 23 Comprehensive Stroke Centres in Poland [1]. Sucesful recanalisation was obtained in almost $65 \%$ of treated cases, with more than half (53\%) having no problems with haemorrhagic transformation. The authors hope that their results will help to harmonise standards for mechanical thrombectomy in Poland.

The second manuscript by Jedrzejczak et al., from 2018, described an expert opinion of the Working Group of the Polish Society of Epileptology on the treatment of convulsive status epilepticus (CSE) in adults and children in Poland [2]. The authors presented the therapeutic options separately for the treatment of different stages of CSE: early preclinical, established, and refractory. Benzodiazepine compounds remain the first line therapeutic option. The report addressed the educational, practical, and organisational aspects of dealing with CSE. The authors hope that their report will improve the therapeutic outcomes of treatment of CSE in Poland.

The third report by Sasiadek et al., also from 2018, presented the second edition of the recommendations of the Polish Medical Society of Radiology for the utilisation of magnetic resonance imaging (MRI) studies in multiple sclerosis (MS) [3]. The protocol proposed the best choices of imaging procedures to obtain standardised MRI studies in this disease. The authors concluded that the neuroimaging monitoring of MS patients with standardised MRI procedures will directly improve the care of patients suffering from MS in Poland.

The final report, by Farkowski et al. from this year, outlined the statements of a Working Group of the Polish National
Consultants in Cardiology and Neurology on the use of implantable cardiac monitors (ICM) for patients after ischaemic embolic stroke of undetermined origin [4]. The authors aimed to provide best practice guidelines for the use of ICM in the management of stroke cases.

The editors of the PJNNS thank these various groups and societies for their interest in publishing in our journal. We hope that our publishing platform will lead to a wider readership, not only by general neurologists and neurosurgeons, but also by other specialists. We also hope, as expressed in these manuscripts, that these position statements and guidelines will indeed lead to the improvement of patient care in Poland.

Zbigniew K. Wszolek, M.D.

Co-Editor-in-Chief

Jarosław Sławek, M.D., Ph.D.

Co-Editor-in-Chief

Łukasz Stolarczyk, M.D.

Administrator

\section{References}

1. Słowik A, Wnuk M, et al. Polish Thrombectomy Initiative. Neurolo-gia i Neurochirurgia Polska. 2017; 51(5): 339-346 doi: 10.1016/j. pjnns.2017.05.004.

2. Jędrzejczak J, Mazurkiewicz-Bełdzińska M, Szmuda M, et al. Convulsive status epilepticus management in adults and children: Report of the Working Group of the Polish Society of Epileptology. Neurologia i Neurochirurgia Polska. 2018; 52(4): 419-426, doi: 10.1016/j. pjnns.2018.04.002.

3. Sąsiadek M, Katulska K, Majos A, et al. Guidelines of the Polish Medi-cal Society of radiology for the routinely used MRI protocol in patients with multiple sclerosis. Neurologia i Neurochirurgia Polska. 2018; 52(6): 638-642, doi: 10.1016/j.pjnns.2018.09.010.

4. Farkowski MM et al., Statement by a Working Group conceived by the Polish National Consultants in Cardiology and Neurology addressing the use of implantable cardiac monitors in patients after ischaemic embolic stroke of undetermined source. Neurologia i Neurochirurgia Polska. 2019; 53(3):181-189, doi: 10.5603/PJNNS.a2019.0018. 